\title{
Interpersonal Arrogance and the Incentive Salience of Power versus
}

\author{
Affiliation Cues \\ Adam K. Fetterman ${ }^{1}$, Michael D. Robinson ${ }^{2}$, and Scott Ode ${ }^{3}$ \\ ${ }^{1}$ Knowledge Media Research Center \\ ${ }^{2}$ North Dakota State University \\ ${ }^{3}$ Medica
}

Note: Correspondence can be sent to Adam Fetterman, Knowledge Media Research Center, Schleichstrasse 6, 72076 Tübingen, Germany. Internet correspondence can be directed to A.Fetterman@iwm-kmrc.de (Phone: +49 7071 979-226; Fax: +49 7071979 200). This publication was made possible by COBRE Grant P20 GM103505 from the Institute of General Medical Sciences (NIGMS), a component of the National Institutes of Health (NIH). Its contents are the sole responsibility of the authors and do not necessarily reflect the official views of NIGMS or NIH. 


\begin{abstract}
The arrogance dimension of the circumplex contrasts people who seemingly value power over affiliation (high arrogance) versus those who do not (low arrogance). Following this line of thinking, and building on an incentive salience model of approach motivation, three studies (total $N=284$ ) examined the differential processing of power versus affiliation stimuli in categorization, perception, and approach-avoidance paradigms. All studies found interactions of the same type. In Study 2, for example, people high in arrogance perceived power stimuli to be larger than affiliation stimuli, but this differential pattern was not evident at low arrogance levels. People high, but not low, in arrogance also approached power stimuli faster than affiliation stimuli in a motor movement task (Study 3). The results contribute to a process-based understanding of how interpersonal arrogance functions while linking such differences to the manner in which power versus affiliation cues are perceived and reacted to.
\end{abstract}

KEYWORDS: Personality, Interpersonal, Arrogance, Power, Affiliation, Incentives, Cues 
Power versus Affiliation 3

\section{Interpersonal Arrogance and the Incentive Salience of Power versus Affiliation Cues}

Taxonomic efforts were necessary to clarify how different personality traits relate to each other and how they group into larger wholes (e.g., the Big 5). As the answers to these questions have become fairly clear, research is needed that seeks to explain how these traits operate as people with them interact with the environment (Pervin, 1994). Two approaches seem particularly promising. One proposes that there is a motivational basis to traits: People differ in their traits because they differ in the types of goals that they have (Carver, Sutton, \& Scheier, 2000). Neurotic people, for example, may be more concerned with the possibility of social rejection than stable people (Denissen \& Penke, 2008). The second approach models interactions with the environment in terms of how presented stimuli are processed and reacted to (Baumert \& Schmitt, 2012; Mathews, 2012). Along these lines, anxious people have been shown to interpret ambiguous stimuli in more threatening terms (Mathews \& MacLeod, 2005).

The two approaches to trait explanation - motivational and cognitive - can be particularly informative when combined. Wilkowski and Robinson (2008), for example, proposed that people low (but not high) in trait anger are motivated to control their hostile thoughts. This led to an information-processing paradigm in which hostile thoughts were primed immediately prior to a task assessing mental control. As hypothesized, people low in trait anger exhibited greater mental control following hostile primes than following neutral primes, but prime type did not matter for people high in trait anger. Wilkowski and Robinson (2008) emphasized the value of their data both for understanding individual differences in anger proneness and for understanding the processes involved. It is possible that other personality traits 
can be similarly decomposed, but doing so will require sustained efforts to bridge the explicitimplicit methodological divide (Robinson \& Wilkowski, in press).

In a sense, the present work did not begin with an effort to understand one personality trait, but rather with the conviction that something important in the trait domain must map onto the differential processing of power versus affiliation stimuli. Power (status, influence) and affiliation (relatedness) figure prominently in our social lives, a point recognized by multiple theorists. Along these lines, Darwin's (1859) initial view of biological fitness emphasized power struggles between organisms, whereas his subsequent view (Darwin, 1871) emphasized affiliation-related adaptations (Miller, 2000). In psychodynamic terms, Horney (1945) considered getting ahead (power) and getting along (affiliation) two of the major challenges social beings must solve (for a more recent analysis, see Hogan, 2007). Bakan (1966) proposed an organizational framework in which agency (closely related to power) and communion (closely related to affiliation) were dominant themes in the lives of individuals, social groups, and even societies (for a more recent exposition, see Trapnell \& Paulhus, 2012). Power and affiliation, finally, are considered two of the prime motivators of social interaction (McClelland, 1987) and dimensions along which people differ (Wiggins \& Trapnell, 1996).

We pitted power versus affiliation incentives against each other in several cognitive paradigms. Our main question was whether tendencies to favor one class of incentives over the other might provide insights into interpersonal features of personality, a possibility suggested by several theoretical frameworks (e.g., Bakan, 1966; Hogan, 2007; Trapnell \& Paulhus, 2012). Before considering this question in further depth, we explain how the paradigms were developed in accordance with cue-related accounts of how motivation operates. 
An important feature of motivation is that it operates on incentives in the environment and likely does so in two ways (McClelland, 1987). First, a given motivation should theoretically prepare us to perceive, react to, and approach stimuli that are motivation-related (Higgins, 1996). For example, a nicotine-deprived smoker should be predisposed to dwell on external smoking cues (Field, Mogg, \& Bradley, 2004). Second, though, cues in the environment can prime related motives (Bargh, Gollwitzer, Lee-Chai, Barndollar, \& Trötschel, 2001). In support of this point, a body of work has shown that incidental motivational cues (e.g., related to achievement) can result in outcomes (such as persistence on a task) that are consistent with stronger levels of that motive (Dijksterhuis \& Aarts, 2010). Together, these top-down and bottom-up influences suggest that important insights about people can be made by studying how incentive cues are processed and reacted to (Robinson \& Berridge, 2003).

To date, cue-processing research has primarily focused on environmental threats and rewards. Following a cognitive-motivational analysis of anxiety (Mogg \& Bradley, 1998), a number of studies have shown that particularly anxious people tend to orient toward environmental threats (MacLeod, 1999). Anxious people might also process threats faster (Augustine, Larsen, \& Lee, 2013) and see threats as larger or more dynamic (Robinson \& Liu, 2013). Just as anxiety orients one toward threats, excitement orients one toward rewards (Tamir \& Robinson, 2007). There is also evidence that rewarding stimuli trigger the approach motivation system (Ode, Winters, \& Robinson, 2012) and that this appears to be particularly true at high levels of extraversion (Robinson, Moeller, \& Ode, 2010). In summary, the cue-processing analysis of approach and avoidance motivation has been a productive one (Harmon-Jones, Price, Peterson, Gable, \& Harmon-Jones, 2013). 
Berridge's (2007) theory of approach motivation is especially germane in thinking about such cue-related processes. Higher levels of approach motivation are thought to increase the salience of reward cues and to bias the organism toward them. This theory has primarily been supported in animal models, but it can be relatively easily translated into cognitive performance predictions. To the extent that a person is motivated to approach a stimulus, that stimulus should be processed faster (Flaisch, Junghöfer, Bradley, Schupp, \& Lang, 2008), should appear larger or closer (Ode et al., 2012), and should speed movements made toward the stimulus (De Houwer, Crombez, Baeyens, \& Hermans, 2001). All such tendencies would presumably support the acquisition of the desirable environmental resource (Dunning \& Balcetis, 2013; Proffitt, 2006). We accordingly focused on speed-based (Study 1), perception-based (Study 2), and movementbased (Study 3) metrics in the present studies.

We sought to extend this cue-processing approach to reactions to power versus affiliation stimuli, particularly in relation to individual differences, but also entertained possible main effects for cue type. People compete for power in a way that they do not compete for affiliation (Kiesler, 1983) and therefore acting quickly in relation to power cues may often be a more pressing issue. People are typically more satisfied with what they have in the affiliation domain than in the power domain (Myers \& Diener, 1995), again considerations that might favor the acquisition of new power-related resources to a greater extent. Finally, power can be seized in a way that affiliation cannot (McClelland, 1987). Front-end cue-related processes might be more biased toward power than affiliation stimuli for this reason. This should be particularly true as levels of interpersonal arrogance increase, however.

Interpersonal Arrogance as a Potential Bias toward Power over Affiliation 
Power and affiliation are interpersonal in the sense that they involve other people in addition to the self (McClelland, 1987). Power is over others and affiliation is with others (McAdams, Hoffman, Mansfield, \& Day, 1996). Interpersonal aspects of personality, in turn, are well captured by what is termed the interpersonal circumplex (Wiggins, 1991). Drawing from theorists such as Sullivan (1953), Leary (1957) detailed the manner in which social features of personality could be appreciated in terms of, and organized by, a circular (or circumplex) arrangement. Wiggins (1979) then developed trait scales to capture individual difference variations in interpersonal style and showed that the scales correlated with each other in exactly the manner suggested by Leary's (1957) analysis (also see Wiggins, Trapnell, \& Phillips, 1988).

Due to its basic and comprehensive nature (Wiggins, 1991), the circumplex has been recommended as a tool for examining new interpersonal phenomena (Gurtman, 1992). Successes along these lines have included interpersonal values (Locke, 2000), social roles (Moskowitz, 1994), social support (Trobst, 2000), personality dysfunction (Alden, Wiggins, \& Pincus, 1990), and nonverbal behavior (Gifford \& O’Connor, 1987). Further, Wiggins and Broughton (1991) showed that 172 diverse scales drawn from personality, social, and clinical psychology could be fit into the interpersonal space defined by the circumplex and circumplex measures have also been used with some success in social cognitive processing studies (Robinson \& Wilkowski, in press). We therefore used the circumplex to characterize the personality features of people who favor power over affiliation in incentive processing.

A further reason for circumplex assessment is that power and affiliation intuitively fit within this interpersonal space. Specifically, higher levels of dominance (versus submission) appear consistent with a power-oriented approach to the social environment and higher levels of warmth (versus coldness) appear consistent with an affiliation-oriented approach to the social 
environment (Wiggins \& Trapnell, 1996). Note, however, that we focus on the differential processing of power versus affiliation cues in our personality-processing designs - for example, we will compare how quickly power words are categorized to how quickly affiliation words are categorized. In this context, arrogance is the dimension of the circumplex that should have the greatest predictive value. This is because arrogance captures an antisocial version of dominance that can be viewed as both power-oriented and low in affiliation (Jones \& Paulhus, 2011; Ruiz, Smith, \& Rhodewalt, 2001). By contrast, the dominance dimension has uncertain relevance to how affiliation stimuli will be processed and the warmth dimension has uncertain relevance to how power stimuli will be processed (Wiggins, 1991; Wiggins \& Trapnell, 1996).

Indeed, the arrogance dimension of the circumplex uniquely captures traits and tendencies of an actively antisocial type. This includes the dark triad traits of narcissism, Machiavellianism, and psychopathy (Furnham, Richards, \& Paulhus, 2013). It also includes traits such as impulsivity, domineeringness, and aggression (Smith, Traupman, Uchino, \& Berg, 2010; Wiggins \& Broughton, 1991). The low end of the arrogance dimension, by contrast, can be viewed in terms of honesty-humility (Furnham et al., 2013) or deference and abasement (Wiggins \& Broughton, 1991). As another way of thinking about the arrogance dimension, one can think of it in terms of unmitigated agency (Rauthmann \& Kolar, 2013), defined in terms of pursuing the self's desires even when doing so will harm others (Helgeson \& Fritz, 1999). These are not only rich conceptions of arrogance, but also ones suggesting that the arrogance dimension, relative to other dimensions of the circumplex, may be particularly predictive of processing tendencies favoring power incentives over affiliation incentives.

In summary, although efforts to bridge the personality-processing divide are scarcer than they should be given their explanatory value, extant knowledge concerning the correlates of 
interpersonal arrogance led us to expect that variations along this continuum would interact with cue type (power versus affiliation) to predict processing performance. At higher levels of arrogance, we hypothesized that speed, perception, and movement would be biased in favor of power cues over affiliation cues. At lower levels of arrogance, we did not expect tendencies of this type. We conducted three studies to examine such interactive hypotheses.

\section{Overview of Studies}

Berridge's (2007) theory of incentive motivation served as a general framework for our predictions. First, to the extent that incentive motivation is high, the organism should be faster to recognize relevant cues in the environment. Study 1 examined this process by assessing the speed with which power versus affiliation stimuli could be categorized. Second, to the extent that incentive motivation is high, relevant cues should gain perceptual salience. Study 2 examined this process by asking individuals to estimate the size of power versus affiliation words. Third, to the extent that incentive motivation is high, approach-related movements should be facilitated. Study 3 examined this process by asking individuals to approach versus avoid stimuli related to power and affiliation using a joystick device. In all cases, interactions between interpersonal arrogance and cue type were expected.

\section{Study 1}

Higher levels of approach motivation are thought to sensitize people to motivationrelevant stimuli (Berridge, 2007). Or, stated in other terms, a dispositional match should result in enhanced accessibility or processing speed (Higgins, 1996). In the first study, accordingly, we used a relatively straightforward choice reaction time paradigm (Robinson, 2004) in which people categorized power versus affiliation stimuli as quickly as possible. An interaction between arrogance and cue type (power versus affiliation) was hypothesized. As a general note, 
all studies used words as stimuli owing to their semantic simplicity and lack of complicating details relative to pictures (Bradley \& Lang, 1999).

\section{Method}

\section{Power Considerations}

Based on our past research on explicit-implicit relations (Robinson \& Wilkowski, in press), we expected medium rather than small or large effect sizes. This past research also guided us to collect sample sizes in the neighborhood of 80 per study. To achieve these sample sizes in approximate terms, we ran each study for a fixed period of time, specified in advance, known to result in similar sample sizes in our past research. All volunteers during this specified time period were processed. Exact sample sizes depended on sign-up and show-up rates, which varied somewhat from study to study. No data analysis occurred until a study's data collection was complete. Post-hoc power analyses revealed power estimates of .86 (Study 1), .83 (Study 2), and .92 (Study 3) to detect interaction effects of the magnitudes obtained.

\section{Participants and Procedures}

Participants in Study 1 were 77 (45 female) undergraduates from North Dakota State University (NDSU) seeking course credit for their psychology classes. They signed up for a generally described personality and cognition study over the Internet using SONA participant registration software. Upon arriving to the laboratory in groups of 6 or less, participants were told they would answer questions about themselves and complete a cognitive task, after which informed consent was obtained. Participants then completed the remainder of the study at personal computers with dividers. The categorization task (see below) was completed before the personality questionnaire to ensure a constant assessment order while guarding against the 
Power versus Affiliation 11

potential priming effects of thinking about one's traits on cognitive performance (Robinson \& Neighbors, 2006). As arrogance is the predictor, though, we describe its assessment first.

\section{Interpersonal Arrogance}

Interpersonal arrogance organizes a number of interpersonally problematic traits such as narcissism and aggression (Wiggins \& Broughton, 1991) and was assessed (via MediaLab software) with the $16 \mathrm{BC}$ (high arrogance) to JK (low arrogance) axis trait adjective markers (e.g., boastful, cocky) of the Interpersonal Adjective Scale-Revised (IAS-R: Wiggins et al., 1988), a well-validated instrument (Wiggins \& Trapnell, 1996). Participants indicated how well $(1=$ extremely inaccurate; $6=$ extremely accurate $)$ each adjective describes the self by moving the mouse to a response button and making a response. After reverse-scoring the $8 \mathrm{JK}$ items, an arrogance score was computed $(M=3.48 ; S D=.80 ;$ alpha $=.81)$.

For discriminant purposes, we also assessed the other three dimensions of the circumplex using the IAS-R. Sixteen adjectives (e.g., jovial, outgoing) assess extraversion $(M=5.03 ; S D=$ .69 ; alpha = .93), 16 (e.g., assertive, dominant $)$ assess dominance $(M=4.41 ; S D=.73$; alpha $=$ $.92)$, and 16 (e.g., coldhearted, unsympathetic) assess coldness $(M=2.01 ; S D=.77$; alpha $=.95)$. As with arrogance, 8 items for each dimension are reverse-scored and predictors constitute averages. While dominance is a theory-relevant predictor of power cue processing, dominance is conceptually independent of coldness (Wiggins, 1991) and therefore not particularly relevant to affiliation cue processing. Vice versa, coldness is a theory-relevant predictor of affiliation cues, but not power cues. In comparison to both, the arrogance dimension may be uniquely predictive in that it captures a hostile form of dominance suggestive of placing power concerns ahead of affiliation concerns, the comparative focus of our paradigms.

\section{Categorization Task}


Participants were asked to quickly and accurately categorize presented words according to whether they signified high levels of power or high levels of affiliation. Instructions further stated that power words imply status and influence, whereas affiliation words imply closeness and relatedness. After consulting several literatures on power and affiliation, we generated a list of 10 power words (affluence, authority, dominance, fortune, money, power, prestige, reputation, status, \& wealth) and 10 affiliation words (affiliation, attachment, belonging, closeness, collaboration, community, cooperation, family, harmony, \& relationships) for use as stimuli. We ensured that the two categories were equal in word frequency $(M=79.85), F<1$, so that results could not be ascribed to this stimulus factor.

An E-Prime program was created to present stimuli and register reaction times. Stimuli were capitalized, randomly selected, and presented at center screen over the course of 120 trials. Responses were made using the 1 and 5 keys of a button box, with response mappings (e.g., $1=$ power or 1 = affiliation) counterbalanced across participants. Labels for the response mappings were continuously presented on the computer screen - below and to the left and right of the stimulus location - to aid the response-mapping process. Errors were penalized with a $1000 \mathrm{~ms}$ visual error message, as is useful when reaction times are the focus of interest. There was a 500 ms blank delay between one response and the presentation of the next stimulus.

Results

\section{Initial Considerations}

Reaction time data were handled in a standard manner. Inaccurate trials were dropped, millisecond values were log-transformed, and log latencies 2.5 SDs faster or slower than the grand mean were replaced with these outlier values (Robinson, 2007). For each participant, we then computed two scores, one averaging across trials involving power words and the other 
averaging across trials involving affiliation words. Performance was very consistent within the power $($ alpha $=.97)$ and affiliation $($ alpha $=.97)$ conditions considered separately. Power versus affiliation difference scores will almost necessarily be less reliable (Miyake, Friedman, Emerson, Witzki, \& Howerter, 2000; Robinson, 2007), but there was some reliability to them (alpha $=.48)$. The latter estimate was computed by subtracting the first-obtained power log time from the firstobtained affiliation log time, the second-obtained power log time from the second-obtained affiliation log time, etc., and then performing an internal consistency analysis on these trial-level difference scores. In the analyses below, we will focus on log-transformed means, but millisecond means are reported for ease of interpretation.

\section{Primary Analysis}

We hypothesized that higher levels of interpersonal arrogance would be linked to enhanced processing for power cues relative to affiliation cues. To examine this interactive hypothesis, we used the General Linear Model (GLM) platform, which is well suited to handle continuous personality dimensions in combination with within-subject designs (Robinson, 2007). The predictors were arrogance (which was z-scored or centered: Aiken \& West, 1991) and cue type (power versus affiliation) and the dependent variable was speed of categorization.

There was a main effect for Arrogance in the paradigm, $F(1,76)=8.61, p<.01$, partial eta squared $(\mathrm{PES})=.10$ (a medium-large effect size $)$. To understand the nature of this main effect, we averaged across the cue type variable and performed a simple regression in which estimated means were calculated for a prototypically low $(-1 S D)$ versus high $(+1 S D)$ level of the arrogance continuum (Aiken \& West, 1991). Categorizations tended to be faster at the high (estimated $M=827 \mathrm{~ms}$ ) relative to low (estimated $M=920 \mathrm{~ms}$ ) level. This is a potentially 
interesting effect that may suggest that arrogant people are "geared to respond", but firmer conclusions of this type would require conceptual replication with other paradigms.

Recall that we entertained the possibility that cue-related processes might generally favor power to affiliation stimuli. This was the case in that there was a main effect for Cue Type, $F(1$, $76)=33.52, p<.01, \mathrm{PES}=.25$, with faster categorization times for power $(M=847 \mathrm{~ms})$ than affiliation $(M=900 \mathrm{~ms})$ stimuli. Although our primary hypothesis pertains to potential interactions with arrogance, this main effect is also interesting, particularly to the extent that it can be conceptually replicated in the paradigms of Studies 2 and 3 .

Of most importance, the interaction between Arrogance and Cue Type was significant, $F(1,76)=5.00, p=.04, \mathrm{PES}=.05$ (a medium effect size). Estimated means for this interaction, at the prototypically low (-1 SD) versus high (+1 SD) levels of interpersonal arrogance, are reported in Figure 1. As shown there, the faster categorization of power than affiliation cues was especially evident as arrogance increased. In other words, there was a systematic relationship between arrogance and the relative accessibility of the two incentive types.

We might stop here, but we wanted to perform some follow-up analyses. Specifically, we wanted to examine the effect of cue type - the within-subject manipulation - at different levels of arrogance. It is possible to do so using the procedures of Wilkowski and Robinson (2007) and covered in more detail by Robinson (2007). First, one turns the within-subject manipulation into a difference score contrasting the two conditions (e.g., affiliation speed minus power speed). Second, one adjusts the personality predictor to reflect low (-1 SD) or high (+1 SD) levels of it (Aiken \& West, 1991). Third, one performs two simple regressions, the first involving the "low" predictor score and the second involving the "high" predictor score. Fourth, one examines inferential statistics for the intercept, which in these regressions reflects how performance varied 
as a function of the within-subject manipulation at a given level of the personality continuum (Wilkowski \& Robinson, 2007). In these follow-up regressions, that is, it is the intercept rather than the slope that is of interest. We present this material to avoid confusion and because followup analyses of this type will be conducted in all studies.

Following these procedures, the within-subject effect of cue type was turned into a difference score in which a person's power latency mean was subtracted from his/her affiliation latency mean, with a higher number meaning faster power cue processing. Then, two simple regressions were performed, one in which the predictor was altered to reflect the low level of arrogance and the other in which it was altered to reflect the high level. As captured by the intercept, the cue type main effect was significant at the high level of arrogance, $t(77)=4.60, p<$ .01 , and reduced in magnitude at the low level, $t(77)=2.56, p=.04$.

Before moving on to other analyses, some additional points should be made. Stimuli for the power and affiliation categories were equal in word frequency, but the affiliation words tended to possess more letters than the power words. This could have contributed to the normative speed difference between the two stimulus categories. Because the task and stimuli were constant across arrogance levels, however, such differences cannot account for the arrogance by cue type interaction that is emphasized.

\section{Accuracy Analysis}

A parallel GLM was performed on accuracy rates. In this analysis, there was no main effect for Arrogance, $F(1,76)=1.52, p=.22$, and there was no Arrogance by Cue Type interaction, $F<1$. Thus, and consistent with a sensitization account (Berridge, 2007), people higher in arrogance appear ready to recognize and respond to power cues (relative to affiliation cues) in a manner that cannot be equated with a speed-accuracy tradeoff. There did appear to be 
a speed-accuracy tradeoff for the cue type main effect, however, in that there was also a Cue Type main effect for accuracy rates, $F(1,76)=13.05, p<.01$, PES = .15: Affiliation words were categorized more accurately $(M=92.10 \%)$ than power words $(M=89.24 \%)$. Our interpretation of the cue type main effect is more provisional, then.

\section{Other Circumplex Dimensions}

Relations among dominance, coldness, and arrogance were as expected (Wiggins, 1979). Coldness and dominance were unrelated, $r(77)=-.17, p=.14$, but arrogance was positively correlated with both coldness, $r(77)=.36, p<.01$, and dominance, $r(77)=.26, p=.02$. Given the relative (power versus affiliation) focus of our paradigm, arrogance seemed the most pertinent predictor. Nonetheless, it was useful to repeat the primary GLM by replacing arrogance with one of the other circumplex dimension scores. There was no personality by cue type interaction for extraversion, $F<1$, or coldness, $F<1$, but there was some trend for dominance, $F(1,76)=3.51, p=.07, \mathrm{PES}=.04$. Estimated means indicated that the pattern of the latter interaction was parallel to that involving arrogance in the sense that the faster categorization of power than affiliation stimuli was more evident at a high $(+1 S D)$ level of dominance (estimated $M \mathrm{~s}=823 \mathrm{~ms} \& 899 \mathrm{~ms})$ than a low $(-1 S D)$ level (estimates $M \mathrm{~s}=873 \mathrm{~ms} \& 903 \mathrm{~ms})$.

The arrogance dimension of the circumplex captures actively antisocial aspects of personality in a parsimonious manner, one not secondary to the dimensions of coldness and dominance (Wiggins \& Trapnell, 1996). For this reason, there seemed no reason to reconfigure the independent dimensions of coldness and dominance to cover the same interpersonal territory. Even so, we explored the possibility that coldness and dominance might interact to predict processing speed. To investigate this possibility, we performed a GLM with cue type, z-scored dominance, z-scored coldness, and all interaction terms as predictors. Rather than reiterating the 
lower-order effects reported above, it is sufficient to say that there was no three-way interaction, $F<1$. Thus, the relative (power versus affiliation) differences observed in Study 1 vary most directly with arrogance (and, to a lesser extent, with dominance).

\section{Participant Sex as a Potential Moderator}

There was a sex difference in arrogance such that men $(M=3.73)$ scored higher than women $(M=3.28), F(1,76)=6.15, p=.02$, PES $=.07$. To determine whether sex moderated the arrogance by cue type interaction emphasized, we reran this GLM with participant sex as an additional factor. The Arrogance by Cue Type by Sex interaction was not significant, $F<1$. Thus, relations between arrogance and differential cue type processing speed were equally strong among men and women.

\section{Discussion and Study 2}

We posited that incentive salience processes would increasingly favor power over affiliation stimuli as levels of interpersonal arrogance increased. We were able to provide support for this idea in the form of an arrogance by cue type interaction involving the relative speeds with which power versus affiliation stimuli could be recognized and categorized. Although accessibility processes of this category speed type have been emphasized by several theories of motivated cognition (as reviewed by Higgins, 1996), categorization speed might not be the best measure of the sort of processes emphasized by Berridge's (2007) incentive salience model of approach motivation. Accordingly, Study 2 extended this analysis to perceptual processes.

When a person is motivated to approach a stimulus, that stimulus should receive a perceptual "boost" by the reward system. Berridge (2007) has found considerable support for this salience-based idea in the context of animal models of motivation (e.g., Wyvell \& Berridge, 2000) and similar phenomena have been demonstrated in both classic (e.g., Bruner \& Goodman, 
1947) and recent (e.g., Veltkamp, Aarts, \& Custers, 2008) work on motivated social cognition (Balcetis \& Lassiter, 2010). Ode et al. (2012) created a paradigm suited to a personality and assessment context in that the measure - font size estimations - is precise and multiple trials can be administered. Consistent with Berridge's (2007) framework, Ode et al. (2012) found that pleasant words were perceived to be larger in font size than neutral or unpleasant words, with no difference between the latter two categories. Study 2 seeks to use this paradigm to examine the relative perceived size of power versus affiliation stimuli. In a pattern paralleling Study 1, we hypothesized greater incentive salience (size overestimations) for power than affiliation stimuli that should be more evident as interpersonal arrogance increases.

Method

\section{Participants, Procedures, and Arrogance Assessment}

A different sample of 86 (44 female) undergraduates from NDSU constituted the Study 2 sample. They too received credit for their psychology classes and registered using SONA software. They also arrived to the lab in groups of 6 or less, received general instructions, and completed the remainder of the study at personal computers with dividers. The perception-based task was administered first and the IAS-R (Wiggins et al., 1988) was given afterwards.

The IAS-R was programmed with MediaLab software and asked people whether 64 interpersonal adjectives were accurate descriptions of the self $(1=$ very inaccurate; $6=$ very accurate). Arrogance was assessed in terms of the $16 \mathrm{BC}$ (positively scored) to JK (reversescored) axis markers (e.g., boastful) of the IAS-R and an average predictor score was computed $(M=3.46 ; S D=.66 ;$ alpha $=.78)$. In addition, the IAS-R dimensions of extraversion $(M=4.88$ $S D=.79 ;$ alpha $=.94)$, dominance $(M=4.33 ; S D=.84 ;$ alpha $=.92)$, and coldness $(M=2.02$; $S D=.76 ;$ alpha $=.93)$ were also assessed. Coldness and dominance were uncorrelated, $r(86)=-$ 
$.02, p=.83$, and arrogance was correlated with coldness, $r(86)=.43, p<.01$, a bit more strongly than with dominance, $r(86)=.21, p=.05$.

\section{Size Estimation Task}

An E-Prime program was created. Participants were told that we were interested in their ability to alternate between two very different tasks. The first would require them to categorize presented words as power-related (implying greater status and influence) or affiliation-related (implying greater closeness and relatedness). This categorization task was to ensure semantic processing of the incentive stimuli, but the procedures (further detailed below) did not allow for precise reaction time measurement in that participants had to move their cursors across significant portions of the computer screen to make their categorizations. Subsequently, participants were to estimate the font size of the presented words, the focus of the paradigm.

In more specific terms, the following events occurred. At the beginning of each paired trial, an array of "Z" letters was presented vertically along the left side of the computer screen. These letters varied in font size from Times New Roman 13 to Times New Roman 23 in 1-point increments. As a counterbalanced variable, some participants were presented with an array that increased in font size down the screen, whereas others received the reversed order of font sizes. This response array remained on the screen during the course of the trials.

After a $1000 \mathrm{~ms}$ delay, a stimulus was randomly selected from the list of 20 words used in Study 1 and placed at center screen. Stimulus words were capitalized to ensure a constant height, but assigned to either a 16 or 20 point Times New Roman font so that it was clear that stimulus sizes differed across trials but in a way that preserved some simplicity to the paradigm. Participants classified each word by moving a centrally presented mouse cursor to one of two boxes at the bottom of the screen, one labeled "power" and the other labeled "affiliation", 
thereupon making a left mouse click. Whether the power box was to the left or right side of the screen was counterbalanced. Participants were given $4000 \mathrm{~ms}$ to categorize each word and the computer program recorded the accuracy of these categorizations. In the case of inaccurate classifications, there was a 2000 ms error message.

Subsequent to a $250 \mathrm{~ms}$ blank delay, the stimulus word was re-presented at center screen in the same font size that occurred at the beginning of the trial. Now, participants were to estimate the font size of the word by clicking on the $\mathrm{Z}$ letter to the left of the screen that was perceived to be of the same font size as the presented word. They were given $3000 \mathrm{~ms}$ to make a size-based perceptual match and cautioned if they were too slow. There were a total of 40 trials such that each of the 20 words was presented in each of the two font sizes.

Results

\section{Initial Considerations}

Perhaps because the trials were somewhat complex, word classification accuracy rates were lower than in Study $1(M=83.03 \%)$. They also differed by Cue Type (power $M=78.83 \%$; affiliation $M=86.28 \%), F(1,85)=20.72, p<.01$, PES $=.33$, but there was no Arrogance main effect involving accuracy rates, $F<1$, nor was there an Arrogance by Cue Type interaction, $F<$ 1. It was deemed best to remove paired trials involving inaccurate stimulus classifications, though the results would be parallel with the inclusion of such trials.

To quantify perceptual size overestimation (or underestimation), we subtracted the actual size of each presented word (16 or 20) from the perceived size of that word (from 13 to 23 ), a difference score in font-size units. Such overestimates were then averaged separately by cue type. Size estimates were fairly consistent within the power $($ alpha $=.91)$ and affiliation $($ alpha $=$ .89) conditions, but the difference score reliability (first-obtained power bias score minus first- 
obtained affiliation bias score, etc.) was lower than desired (alpha $=.31)$. This can likely be rectified in future studies by the provision of more trials. As was true in the Ode et al. (2012) paper, there was a general tendency toward size overestimation for both cue types (see below for means), $p s<.01$, results that make sense from an incentive salience perspective (i.e., people generally seek both power and affiliation to some extent: McClelland, 1987).

\section{Primary Analysis}

In a GLM analysis, we examined size overestimations as a function of both cue type and Z-scored levels of interpersonal arrogance (Robinson, 2007). There was no main effect for Arrogance, $F<1$, nor was one expected. Study 1 found that power stimuli were classified faster than affiliation stimuli. We thought it possible that the former class of stimuli would also appear larger. This was the case in that there was a Cue Type main effect, $F(1,85)=6.31, p<.01$, PES $=.08(M \mathrm{~s}=1.48 \& 1.30$ font size overestimation units for power $\&$ affiliation stimuli, respectively). This very interesting normative effect, however, was qualified by the hypothesized Arrogance by Cue Type interaction, $F(1,85)=4.37, p<.01$, PES $=.05$ (a medium effect size).

Estimated means for the significant interaction, at a prototypically low (-1 SD) versus high (+1 SD) level of arrogance, are displayed in Figure 2. As shown there, the tendency to overestimate the size of power relative to affiliation cues appeared particular to highly arrogant individuals. To confirm this visual interpretation, we performed follow-up analyses parallel to Study 1. Specifically, we converted the within-subject cue type main effect into a difference score (power overestimations minus affiliation overestimations). We then performed two simple regressions, one in which the predictor reflected a high (+1 SD) level of arrogance and the other in which it reflected a low (-1SD) level. Of interest were statistics for the intercept, which in this case reflect the influence of the cue type manipulation at a given level of arrogance (Robinson, 
2007; Wilkowski \& Robinson, 2007). The intercept was significant in the first simple regression, $t(86)=3.34, p<.01$, but not in the second, $t(86)=0.38, p=.70$. Thus, a differential bias favoring power cues was evident only as levels of interpersonal arrogance became high.

\section{Other Circumplex Dimensions}

Three additional GLMs were run in which arrogance was replaced by extraversion, dominance, or coldness, the other IAS-R dimensions. Personality by cue type interactions were not evident for extraversion, $F<1$, or dominance, $F(1,83)=1.50, p=.22$, but there was a Coldness by Cue Type interaction, $F(1,83)=6.69, p=.01, \mathrm{PES}=.08$. The pattern of the latter interaction paralleled arrogance in that the tendency to perceive power stimuli to be larger than affiliation stimuli was more pronounced at a high $(+1 S D)$ level of coldness (estimated $M \mathrm{~s}=1.60$ $\& 1.24$ font size overestimation units) than a low $(-1 S D)$ level (estimated $M \mathrm{~s}=1.39 \& 1.39)$.

As in Study 1, we then sought to determine whether dominance and coldness interacted to predict the effects of cue type. To investigate this question, a GLM involving z-scored dominance, z-scored coldness, and their interaction was performed. Lower-order effects were parallel to those already reported and the three-way interaction was not significant, $F<1$. Accordingly, the tendency to see power stimuli as larger than affiliation stimuli aligns itself relatively directly with arrogance (and, in Study 2, with coldness).

\section{Participant Sex as a Potential Moderator}

As might be expected, men $(M=3.66)$ scored higher in arrogance than women $(M=$ 3.24), $F(1,84)=9.83, p<.01$, PES $=.10$. In addition, a GLM revealed that participant sex acted as a moderator in that there was an Arrogance by Cue Type by Sex interaction, $F(1,81)=8.81, p$ $<.01, \mathrm{PES}=.10$. Estimated means indicated that the arrogance by cue type interaction was pronounced among men (estimated $M \mathrm{~s}=1.15,1.23,1.69, \& 0.94$ for low arrogant/power, low 
arrogant/affiliation, high arrogant/power, \& high arrogant/affiliation combinations, respectively), but not women (estimated $M \mathrm{~s}=1.55,1.34,1.71, \& 1.54)$. It is intriguing that incentive salience processes appeared to favor affiliation cues to a greater extent among arrogant women than among arrogant men, but similar sex-related moderation effects were not observed in Studies 1 or 3. Nonetheless, further work on these sorts of interactions may be of interest.

\section{Discussion and Study 3}

Size overestimations have been shown to track reward value (Ode et al., 2012; Veltkamp et al., 2008), as they should from an incentive salience view of approach motivation (Berridge, 2007). It was therefore informative that there was a significant tendency for people to think that power-related stimuli were larger than affiliation-related stimuli. Of perhaps more importance, this perceptual bias was particularly if not exclusively evident at a high level of interpersonal arrogance, though the reliability of the paradigm (in terms of difference scores) was less than desired. In any case, the results of Study 2 converge with those of Study 1 in suggesting that as arrogance increases, so does the incentive salience of power to affiliation cues.

We sought to extend this analysis in Study 3. Lewin (1936) proposed that approach motivation should speed movements toward an incentive and avoidance motivation should speed movements away from a disincentive. Movement dynamics of this type were confirmed in Miller's (1944) animal research and subsequently in a literature asking human beings to move joysticks either toward or away from valenced stimuli (for a review, see Krieglmeyer \& Deutsch, 2010). A joystick paradigm was created to examine such movement-related processes. For comparative purposes, and to ensure stimulus processing, every trial presented both power and affiliation cues, but at different locations of a computer screen. Movement instructions emphasized approaching versus avoiding stimuli to encourage this conception of the movements 
(Krieglmeyer \& Deutsch, 2010). Incentive motivation processes should favor both approaching a desired stimulus and disfavor avoiding it (Berridge, 2007; Lewin, 1936). For this reason, a comparison of approach versus avoidance conditions was a key factor. The hypothesis was that as interpersonal arrogance increases, the approach-avoidance comparison should increasingly favor power to affiliation - i.e., there should be a 3-way interaction.

Joystick movement paradigms can be a bit noisy, however. Block instructions will fade in and out of prominence in working memory, readiness for stimuli will waver, and movementrelated mappings will often have to be updated, contributing further noise (Duncan et al., 2000). These non-motivational processes will primarily contribute to movement onset times (Jensen, 2006). By contrast, both classic and modern theories of approach and avoidance motivation suggest that it should be particularly evident in movement-related processes (Elliot, 2006) and there are data in support of this idea. For example, Robinson, Zabelina, Boyd, Bresin, and Ode (in press) found that people moved the symbolic self faster toward positive than negative locations, but that this self-favoring pattern was not evident for movement onsets. Similarly, Boyd, Robinson, and Fetterman (2011) found movement times to be sensitive to motivational conflicts in a way that movement onset times were not. For both theoretical (Carver et al., 2000; Elliot, 2006) and practical (Duncan et al., 2000; Jensen, 2006) reasons, then, our hypotheses were specific to movement times relative to movement onset times.

\section{Method}

\section{Participants, Procedures, and Arrogance Assessment}

Participants in Study 3 were 118 (67 female) undergraduates from NDSU who received psychology course credit. They were given general instructions parallel to Studies 1 and 2 and then seated at one of 6 private computers. Arrogant interpersonal functioning was again assessed 
using the $16 \mathrm{BC}$ to JK axis adjective markers of the IAS-R $(M=3.49 ; S D=.82 ;$ alpha $=.90)$. To locate Study 3's approach-avoidance tendencies within the circumplex, we also assessed IAS-R extraversion $(M=4.90 ; S D=.79 ;$ alpha $=.95)$, coldness $(M=1.96 ; S D=.72 ;$ alpha $=.95)$, and dominance $(M=4.28 ; S D=.79 ;$ alpha $=.91)$. Coldness and dominance were uncorrelated, $r(118)=-.10, p=.26$, whereas arrogance was positively correlated with both coldness, $r(118)=$ $.39, p<.01$, and dominance, $r(118)=.35, p<.01$.

Approach-Avoidance Task

Each of the 160 trials of the approach-avoidance task (programmed with E-Prime software) presented two words on the screen. One was a power word and the other was an affiliation word. The words were randomly selected and paired from the lists presented in the Study 1 method section. One was presented 1.60 inches from the top of the computer screen and the other was presented 1.60 inches from the bottom of the computer screen. The computer screen height was 13.65 inches. Whether the power word of each pair was toward the top or bottom of the screen was randomized across trials.

There were four block types, respectively asking individuals to approach power stimuli, approach affiliation stimuli, avoid power stimuli, or avoid affiliation stimuli. In approach conditions, participants were to move a Saitek Aviator-01 Dual Throttle joystick toward the incentive indicated. This movement should be easier if a stimulus is desired. In avoidance conditions, they were to move the joystick away from the incentive indicated. This movement should be more difficult if a stimulus is desired. As an example, the power-approach condition asked participants to move the joystick cursor toward the top (bottom) of the computer screen if a power word was in a high (low) vertical position. Block types were repeated in four consecutive randomized orders for a total of 16 blocks, each involving 10 trials. 
Trial procedures were as follows. The joystick cursor first appeared at center screen. After a $400 \mathrm{~ms}$ blank delay, the word pair for the trial was presented. Participants were asked to quickly and accurately move the joystick cursor - which remained visible and tracked the path made by the participant - up or down on the basis of block instructions and, in either case, to contact the top or bottom of the computer screen to terminate the trial. If a movement was initiated in the wrong direction for a given trial, participants received a $1000 \mathrm{~ms}$ visual error message informing them that they were moving in the wrong direction. Correct movements were followed by a "please return to center" instruction. Upon returning to center screen, there was a 100 ms blank delay before the next trial in a block started.

Onset was defined in terms of moving the joystick cursor .33 inches in the correct direction following stimulus presentation. Movement time was the time that elapsed between this onset and the completion of the trial movement. As all trials involved traversing the same spatial distance, these movement times can be considered (motivation-infused) velocities. Our hypotheses were specific to the movement time measure.

\section{Results}

\section{Initial Considerations}

Trials associated with incorrect movement directions were first dropped. Positive skew was evident for both onset and movement time distributions and therefore both distributions were log-transformed. Subsequently, and separately so for each distribution, $2.5 S D$ outlier values were replaced (i.e., the same transformation procedures used in Study 1). Log latency means were then averaged for cells of the 2 (approach versus avoidance) by 2 (power versus affiliation) within-subject design. Analyses focused on these log-transformed values but millisecond means will be reported for the sake of interpretation ease. 
There were no hypotheses involving accuracy rates (i.e., movements in the correct direction), but they were analyzed for the sake of complete reporting and to evaluate the possibility of speed-accuracy tradeoffs. This GLM analysis, like that concerning movement times, included the two within-subject factors of cue type (power versus affiliation) and movement direction (approach versus avoid) in addition to z-scored variations in interpersonal arrogance. All $F$-values were less than 1, save for a main effect for Movement Direction, $F(1$, $116)=18.73, p<.01, \mathrm{PES}=.14$. Not surprisingly given the desirability of the incentives, approach movements tended to be more accurately initiated $(M=91.64 \%)$ than avoidance movements $(M=89.72 \%)$.

We were particularly interested in movement times, but an initial GLM did examine movement onset times. In this onset-based analysis, there was a main effect for Movement Direction, $F(1,116)=54.54, p<.01$, PES $=.32$, with onsets for approach $(M=857 \mathrm{~ms})$ faster than onsets for avoidance $(M=939 \mathrm{~ms})$. This main effect depended on the incentive under consideration, however, in that there was a Cue Type by Movement Direction interaction, $F(1$, 116) $=10.09, p<.01, \mathrm{PES}=.08$. The interaction was conceptually parallel to the cue type main effects of Studies 1 and 2. Specifically, approach onsets were faster for power $(M=839 \mathrm{~ms})$ than affiliation $(M=875 \mathrm{~ms})$ stimuli, whereas avoidance onsets were slower for power $(M=950 \mathrm{~ms})$ than affiliation $(M=928 \mathrm{~ms})$ stimuli. There was no Cue Type main effect, $F<1$, nor were there main effects or interactions involving Arrogance, $p \mathrm{~s}>.45$. Significant results involving interpersonal arrogance, then, will be located in the theory-emphasized (Boyd et al., 2011; Miller, 1944) movement phase of the joystick task.

\section{Primary Analysis}


The primary GLM analysis examined movement times as a function of cue type, movement direction, and interpersonal arrogance. We first concentrate on potential normative effects - i.e., those that collapse across arrogance levels. Main effects for Cue Type and Movement Direction were not significant, $F_{\mathrm{S}}<1$, but there was some tendency toward an interaction between these factors, $F(1,116)=3.40, p=.08, \mathrm{PES}=.03$. The nature of the interaction was parallel to that for onset times. Specifically, approach movements were somewhat faster for power $(M=201 \mathrm{~ms})$ than affiliation $(M=205 \mathrm{~ms})$ stimuli, but avoidance movements were ever so slightly slower for power $(M=201 \mathrm{~ms})$ than affiliation $(M=200 \mathrm{~ms})$ stimuli. Movement times were quite consistent within each of the four conditions (alphas $=.96-$ .97) and the internal reliability of difference scores of difference scores (difference score $1=$ (first-obtained power/avoid log time + first-obtained affiliation/approach log time) - (firstobtained power/approach log time + first-obtained affiliation/avoid log time)) was higher (alpha $=.53$ ) than one might expect for scores of this type (Miyake et al., 2000; Robinson, 2007).

With respect to the individual difference component of the design, there was no main effect for Arrogance, $F<1$. Thus, a tendency toward speedier movements in general did not characterize arrogant individuals in this paradigm. There was no Arrogance by Cue Type interaction, $F<1$, nor should there be as this interaction collapses across the critically important approach-avoidance manipulation. There was also no Arrogance by Movement Direction interaction, $F<1$. Thus, and consistent with the findings of Studies 1 and 2, incentive type will be important in understanding the cue-related processes that differ by arrogance.

Instead, and as hypothesized, there was a significant Arrogance by Cue Type by Movement Direction three-way interaction, $F(1,116)=4.87, p=.03$, PES $=.04$ (in between a small and medium effect size). To break down the three-way interaction, follow-up analyses 
were performed as in Studies 1 and 2. We first sought to determine whether cue type and movement direction (the two within-subject factors) interacted to predict performance at a given level of arrogance (either high or low). To investigate this question, we computed a difference score to reflect the two-way interaction: ((approach/affiliation + avoid/power) - (approach/power + avoid/affiliation)). Higher numbers indicate favoring the approach, and disfavoring the avoidance, of power incentives relative to affiliation incentives. We then performed two simple regressions predicting this difference score, one for a high $(+1 S D)$ level of arrogance and the other for a low $(-1 S D)$ level. It is the intercepts rather than slopes that are of interest in these regressions (Robinson, 2007; Wilkowski \& Robinson, 2007).

At the high level of arrogance, the intercept reflecting the cue type by movement direction interaction was significant, $t(118)=2.52, p=.01$. Estimated means for highly arrogant individuals are displayed in Figure 3 and they indicate that arrogant people were faster to approach power than affiliation incentives as well as slower to avoid power than affiliation incentives. Approach-avoidance processes, that is, favored power over affiliation at high levels of arrogance. At the low level of arrogance, the difference score was not significant, $t(118)=-$ 0.97, $p=.33$ (estimated $M \mathrm{~s}=208 \mathrm{~ms}, 199 \mathrm{~ms}, 208 \mathrm{~ms}, \& 206 \mathrm{~ms}$ for approach/power, approach/affiliation, avoid/power, \& avoid/affiliation conditions, respectively). Thus, the performance of low-arrogance individuals did not favor an incentive category, results in line with Studies 1 and 2 in the context of a different paradigm.

One might expect arrogance to interact with cue type primarily with respect to approach movements. To discern whether this was the case, two further difference scores were computed. One quantified the extent to which avoidance movement times were slower when power incentives were involved (i.e., avoid/power - avoid/affiliation). Levels of interpersonal arrogance 
did not predict this difference score in a simple regression, $t(118)=-1.05, p=.30$. The other difference score quantified the extent to which approach movement times were faster when power incentives were involved (i.e., approach/affiliation - approach/power). Arrogance was a significant predictor of this difference score, $t(118)=2.34, p=.02$. Furthermore, differential approach movement speed (as reflected in the difference score intercept: Wilkowski \& Robinson, 2007) was significant at a high $(+1 S D), t(118)=2.18, p=.03$, but not low $(-1 S D)$, $t(118)=-1.14, p=.26$, level of interpersonal arrogance. These follow-up analyses converge on the approach-related nature of the findings.

\section{Other Circumplex Dimensions}

For discriminant purposes, we also performed analyses in which interpersonal arrogance was replaced with one of the other three dimensions of the circumplex - extraversion, coldness, or dominance. The dependent measure was movement times and the within-subject design included the cue type and movement direction factors. In contrast to the analysis involving arrogance, three-way interactions for extraversion, $F<1$, coldness, $F(1,116)=2.01, p=.16$, and dominance, $F<1$, were not significant. In an additional analysis, there was also no four-way cue type by movement direction by coldness by dominance interaction, $F<1$. Thus, differential reactivity to power stimuli relative to affiliation stimuli aligns itself somewhat uniquely with the arrogance dimension of the circumplex. Such results make sense. For example, dominance can be enacted in either a warm or cold manner, whereas arrogance captures a cold or hostile form of dominance more pertinent to our comparative (power $>$ affiliation) focus.

\section{Participant Sex as a Potential Moderator}

As in Studies 1 and 2, males scored higher in arrogance $(M=3.85)$ than females $(M=$ $3.23), F(1,115)=19.10, p<.01, \mathrm{PES}=.14$. Arrogance functioned similarly in males and 
Power versus Affiliation 31

females, however, as the significant (arrogance by cue type by movement direction) three-way interaction of primary interest was not moderated by participant sex in a follow-up GLM, $F(1$, $113)=1.74, p=.19$. Overall, then, we emphasize the generality of the arrogance-linked processes identified by our studies.

\section{Discussion}

The goal of Study 3 was to focus somewhat directly on implicit approach-avoidance processes. We were able to do so by creating a joystick paradigm such that the speed with which movements were made toward and away from power incentives as well as toward and away from affiliation incentives could be compared. As hypothesized, the prototypically arrogant person favored power over affiliation incentives in this paradigm, whereas the prototypically nonarrogant person did not. In addition, a further breakdown of the findings revealed that such dynamics were particularly evident for approach movements. In sum, a good deal of evidence was offered for the idea that incentive salience and reactivity processes increasingly favor power over affiliation cues as levels of interpersonal arrogance increase. These results provide insights into how the personality trait dimension of arrogance functions.

\section{General Discussion}

Power and affiliation are key social motives, motives that are frequently activated by relevant environmental incentives (McClelland, 1987). We sought to compare such activation processes and did so in paradigms that should be sensitive to how the approach system operates (Berridge, 2007). The results are suggestive of differences in the manner in which power and affiliation cues are processed and relatively more definitive concerning a role for interpersonal arrogance in this cue-related context. Each set of findings is discussed in turn, albeit more tentatively in the former case than the latter. 
Power versus Affiliation 32

Incentive Processes Involving Power versus Affiliation

Cognitive-motivational processes should be particularly attuned to opportunities that can be quickly gained or lost (Bechara, 2004; Wurm \& Vakoch, 2000). There are reasons for thinking that power may be different than affiliation in this respect. Whereas affiliation builds up somewhat gradually, power can wax and wane quite quickly on the basis of components and features of the social environment (Kiesler, 1983). In addition, there is a competitiveness to power that is not present in affiliation (Moskowitz, 1994) and, for this reason too, we entertained the possibility that incentive salience processes might favor a more immediate appraisal and response to power cues than to affiliation cues.

In the context of such considerations, it is useful to take stock of the normative findings. When collapsing across arrogance levels, Study 1 found that power stimuli were categorized faster than affiliation stimuli. For this normative effect, though, there was some evidence of a speed-accuracy tradeoff. In addition, and although word frequency was equivalent for the two incentive categories, word length tended to be longer for the affiliation category, which could have affected categorization speed. Study 2 found that power stimuli were overestimated in font size to a greater extent than affiliation stimuli, a relatively straightforward result in suggesting the greater incentive salience of power cues. In Study 3, though, the cue type by movement direction interaction of greatest interest did not reach conventional levels of significance. Overall, then, the normative effects were somewhat consistent, but not entirely free of qualifications. Of more importance, though, such normative trends interacted with interpersonal arrogance and it is the latter interactions that should be more greatly emphasized.

Interpersonal Arrogance from a Cue-Processing Perspective 
Many of the correlates of interpersonal arrogance - such as narcissism, aggression, and impulsiveness (Furnham et al., 2013; Wiggins \& Broughton, 1991) - are suggestive of an orientation to the social environment in which power concerns may be weighted more heavily than affiliation concerns. The present research sought to support this motivational perspective on arrogance while tying it to a biological theory of approach-related processes (Berridge, 2007). Arrogant people, by this analysis, are those whose incentive motivation systems are particularly sensitized to power rather than affiliation cues in the environment.

Findings supported this process-based analysis of individual differences in arrogance: The highly arrogant person categorized power stimuli more quickly than affiliation stimuli, thought power stimuli were larger, and were biased to approach power stimuli with greater vigor. These same differential tendencies were greatly attenuated or absent at low levels of arrogance. Interactions of this type were generally of a moderate effect size, which is characteristic for successful bridges of the personality-processing interface (Robinson \& Wilkowski, in press). In any case, we suggest that the findings help us understand the antisocial tendencies of arrogant people in that incentive motivation processes favoring power to affiliation cues would be precisely those that might lead arrogant people to pursue power opportunities even at the expense of their personal relationships (Rauthmann \& Kolar, 2013).

Viewed from another perspective, the findings highlight the utility of focusing on the interpersonal arrogance dimension of the circumplex for certain research and predictive purposes. Although it might be tempting to think of the arrogance axis of the circumplex as somehow less fundamental than the dominance or coldness ones, this is not the case. As a circumplex, the space is equally well characterized in terms of arrogance and extraversion - two forms of social activation - as it is by dominance and coldness (Gurtman, 2009). Further, to the 
extent that one is interested in hostile (or dark) forms of agency, this is the circumplex dimension that one should focus on (Wiggins \& Broughton, 1991). To such considerations, we add that interpersonal arrogance should be of particular interest when phenomena can be viewed in terms of prioritizing power over affiliation in transactions with the social environment.

Although the results were consistent, they can be extended. We showed that cue-related processes favoring power to affiliation varied most directly with arrogance relative to other dimensions of the interpersonal circumplex (e.g., extraversion). And while the interpersonal circumplex is comprehensive in certain ways, it is not comprehensive in others. Whether the present cue-related processes vary by neuroticism or openness to experience, for example, cannot be known by adopting a circumplex framework (Wiggins \& Trapnell, 1996). Additionally, we focused on arrogance because it captures what is common to multiple "dark" traits like narcissism, psychopathy, and aggression (Wiggins \& Broughton, 1991). One might want to make distinctions among these traits, however (Jones \& Paulhus, 2011), and doing so would require additional study. It would also be useful to determine whether implicit motives (McClelland, 1987) are predictive of the processes examined. In short, there is further work to do in characterizing the personality side of power-favoring implicit tendencies.

We were consistent in our focus on personality-processing relationships. That is, all studies sought to link variations in arrogance-related traits (as assessed by the IAS-R) to differential processing for power cues versus affiliation cues. This consistency of focus can be considered advantageous in that it builds on a standardized approach to personality assessment in locating the processes involved (Robinson \& Gordon, 2011). Having established these personality-processing relationships, though, it can now be determined whether the processing biases identified can be used to predict actual social behaviors of an arrogance-related type. For 
example, it is plausible (but not shown) that power-favoring individuals will have more antagonistic personal relationships (Smith et al., 2010), in part because they react to provocation more aggressively (Wilkowski \& Robinson, 2010). There is also the possibility that arrogance/outcome relationships might be mediated by the processing tendencies highlighted (Robinson \& Wilkowski, in press). We must emphasize, though, that it is desirable to be programmatic in personality-processing research (Robinson \& Gordon, 2011). From this perspective, the present findings build a foundation for the outcome-focused work of the future.

\section{Questions and Further Considerations}

Should the results be understood in motivational terms? Although the Study 1 paradigm might be ambiguous in this regard, the paradigms used in Studies 2 and 3 are straightforward translations of Berridge's (2007) well-validated incentive theory of approach motivation. In addition, motivational interpretations of motor movements in approach-avoidance tasks have been increasingly supported (Krieglmeyer \& Deutsch, 2010; Krieglmeyer, Deutsch, De Houwer, \& De Raedt, 2010). Thus, while recognizing the relatively cognitive nature of the paradigms, we nonetheless suggest that motivational factors were involved.

Might motivation be conceptualized in terms of processes other than those assessed? There is no doubt that this is the case. For example, none of the paradigms asked people about their goals or measured the persistence with which incentives were pursued. Accordingly, the findings need to be understood in relatively implicit cognitive terms. In fact, although cue-related processes seemed to favor power to affiliation stimuli in the normative findings, self-reports of motivation might suggest the reverse (Kasser \& Ryan, 1993). A contrast of these two sorts of measures might be an interesting direction for future research. 
Stimuli were desirable (e.g., prestige, closeness) rather than undesirable in nature. This was a necessary feature of the paradigms in that we sought to focus on approach-related processes. Would interpersonal arrogance also interact to predict processing tendencies exhibited for deficit cues to power (e.g., poor) versus affiliation (e.g., lonely)? On the one hand, this seems somewhat likely. On the other hand, our results do not speak to this question. Accordingly, future research might employ the latter types of cues as a way of extending the present analysis.

The success of the present studies likely consisted in part of pitting power cues against affiliation cues. This is appropriate in that interpersonal arrogance should matter quite a bit under circumstances in which pursuing one class of incentives is done while risking the other class, such as in arguing with a friend (Smith, Glazer, Ruiz, \& Gallo, 2004). Furthermore, a contrast of processes involving these incentives seems apt given their centrality to social interaction and it is not clear what an informative control condition might otherwise consist of. Nonetheless, the results need to be understood in terms of the comparative designs that were instantiated.

Finally, we note that incentive salience theory encompasses perceptual, cognitive, and motoric components (Berridge, 2007). To flesh out this theory, it seemed important to develop a paradigm for each component (Study $1=$ cognitive; Study $2=$ perceptual; Study $3=$ motoric). As a consequence of doing so, however, the studies should be considered conceptual replications rather than direct ones. To have greater confidence in any one set of findings, direct replications, preferably with larger sample sizes, may be considered necessary. This point should be underlined given the novelty of the results. In addition, it would be informative to assess all three sets of processes among the same set of participants. Doing so would enable one to determine whether, for example, people who see a class of incentives as larger are also people who 
approach that class of incentives faster in movement-related terms. Incentive salience theory (Berridge, 2007) should predict correlations of this type, but they are as yet unexamined.

\section{Conclusions}

We hypothesized that incentive motivation processes would favor power to affiliation cues to a greater extent as levels of interpersonal arrogance increased. Consistent findings of this type occurred and they contribute to a cognitive-motivational understanding of how interpersonal arrogance functions. The findings also encourage future efforts to apply incentive motivation theories to the manner in which people differ from each other. 


\section{References}

Aiken, L., \& West, S. (1991). Multiple regression: Testing and interpreting interactions. Thousand Oaks, CA: Sage Publications, Inc.

Alden, L. E., Wiggins, J. S., \& Pincus, A. L. (1990). Construction of circumplex scales for the Inventory of Interpersonal Problems. Journal of Personality Assessment, 55, 521-536.

Augustine, A. A., Larsen, R. J., \& Lee, H. (2013). Affective personality traits and cognition: Interactions between extraversion/neuroticism, affect, and cognition. In: M. D. Robinson, E. R. Watkins, \& E. Harmon-Jones (Eds.), Handbook of cognition and emotion (pp. 312328). New York: Guilford Press.

Bakan, D. (1966). The duality of human existence: An essay on psychology and religion. Oxford, England: Rand McNally.

Balcetis, E., \& Lassiter, G. D. (2010). Social psychology of visual perception. New York: Psychology Press.

Bargh, J. A., Gollwitzer, P. M., Lee-Chai, A., Barndollar, K., \& Trötschel, R. (2001). The automated will: Nonconscious activation and pursuit of behavioral goals. Journal of Personality and Social Psychology, 81, 1014-1027.

Baumert, A., \& Schmitt, M. (2012). Personality and information processing. European Journal of Personality, 26, 87-89.

Bechara, A. (2004). A neural view of the regulation of complex cognitive functions by emotion. In: P. Philippot \& R. S. Feldman (Eds.), The regulation of emotion (pp. 3-32). Mahwah, NJ: Lawrence Erlbaum Associates.

Berridge, K. C. (2007). The debate over dopamine's role in reward: The case for incentive salience. Psychopharmacology, 191, 391-431. 
Boyd, R. L., Robinson, M. D., \& Fetterman, A. K. (2011). Miller (1944) revisited: Movement times in relation to approach and avoidance conflict. Journal of Experimental Social Psychology, 47, 1192-1197.

Bradley, M. M., \& Lang, P. J. (1999). Affective norms for English words (ANEW): Instruction manual and affective ratings. Center for Research in Psychophysiology. Gainesville, FL: University of Florida.

Bruner, J., \& Goodman, C. (1947). Value and need as organizing factors in perception. The Journal of Abnormal and Social Psychology, 42, 33-44.

Carver, C. S., Sutton, S. K., \& Scheier, M. F. (2000). Action, emotion, and personality: Emerging conceptual integration. Personality and Social Psychology Bulletin, 26, 741751.

Darwin, C. R. (1859). The origin of species. London, England: Murray.

Darwin, C. R. (1871). The descent of man, and selection in relation to sex. London, England: Murray.

De Houwer, J., Crombez, G., Baeyens, F., \& Hermans, D. (2001). On the generality of the affective Simon effect. Cognition and Emotion, 15, 189-206.

Denissen, J. J. A., \& Penke, L. (2008). Motivational individual reaction norms underlying the Five-Factor model of personality: First steps towards a theory-based conceptual framework. Journal of Research in Personality, 42, 1285-1302.

Dijksterhuis, A., \& Aarts, H. (2010). Goals, attention, and (un)consciousness. Annual Review of Psychology, 61, 467-490.

Duncan, J., Seitz, R. J., Kolodny, J., Bor, D., Herzog, H., Ahmed, A., et al. (2000). A neural basis for general intelligence. Science, 289, 457-460. 
Dunning, D., \& Balcetis, E. (2013). Wishful seeing: How preferences shape visual perception. Current Directions in Psychological Science, 22, 33-37.

Elliot, A. J. (2006). The hierarchical model of approach-avoidance motivation. Motivation and Emotion, 30, 111-116.

Field, M., Mogg, K., \& Bradley, B. P. (2004). Eye movements to smoking-related cues: Effects of nicotine deprivation. Psychopharmacology, 173, 116-123.

Flaisch, T., Junghöfer, M., Bradley, M. M., Schupp, H. T., \& Lang, P. J. (2008). Rapid picture processing: Affective primes and targets. Psychophysiology, 45, 1-10.

Furnham, A., Richards, S. C., \& Paulhus, D. L. (2013). The dark triad of personality: A 10 year review. Social and Personality Psychology Compass, 7, 199-216.

Gifford, R., \& O’Connor, B. (1987). The interpersonal circumplex as a behavior map. Journal of Personality and Social Psychology, 52, 1019-1026.

Gurtman, M. B. (1992). Construct validity of interpersonal personality measures: The interpersonal circumplex as a nomological net. Journal of Personality and Social Psychology, 63, 105-118.

Gurtman, M. B. (2009). Exploring personality with the interpersonal circumplex. Social and Personality Psychology Compass, 3, 601-619.

Harmon-Jones, E., Price, T. F., Peterson, C. K., Gable, P. A., \& Harmon-Jones, C. (2013). The influence of behavioral approach and behavioral inhibition sensitivities on emotive cognitive processes. In: M. D. Robinson, E. R. Watkins, \& E. Harmon-Jones (Eds.), Handbook of cognition and emotion (pp. 329-346). New York: Guilford Press 
Helgeson, V. S., \& Fritz, H. L. (1999). Unmitigated agency and unmitigated communion: Distinctions from agency and communion. Journal of Research in Personality, 33, 131158.

Higgins, E. T. (1996). Knowledge activation: Accessibility, applicability, and salience. In E. T. Higgins \& A. W. Kruglanski (Eds.), Social psychology: Handbook of basic principles (pp. 133-168). New York: Guilford.

Hogan, R. (2007). Personality and the fate of organizations. Mahwah, NJ: Erlbaum.

Horney, K. (1945). Our inner conflicts. Oxford, England: Norton \& Co.

Jensen, A. R. (2006). Clocking the mind: Mental chronometer individual differences. Amsterdam, Netherlands: Elsevier.

Jones, D. N., \& Paulhus, D. L. (2011). Differentiating the Dark Triad within the interpersonal circumplex. In: L. M. Horowitz \& S. Strack (Eds.), Handbook of interpersonal psychology: Theory, research, assessment, and therapeutic interventions (pp. 249-267). Hoboken, NJ: John Wiley \& Sons.

Kasser, T., \& Ryan, R. M. (1993). A dark side of the American dream: Correlates of financial success as a central life aspiration. Journal of Personality and Social Psychology, 65, 410-422.

Kiesler, D. J. (1983). The 1982 Interpersonal Circle: A taxonomy for complementarity in human transactions. Psychological Review, 90, 185-214.

Krieglmeyer, R., \& Deutsch, R. (2010). Comparing measures of approach-avoidance behavior: The manikin task vs. to versions of the joystick task. Cognition and Emotion, 24, 810828. 
Krieglmeyer, R., Deutsch, R., De Houwer, J., \& De Raedt, R. (2010). Being moved: Valence activates approach-avoidance behavior independently of evaluation and approachavoidance intentions. Psychological Science, 21, 607-613.

Leary, T. (1957). Interpersonal diagnosis of personality: A functional theory and methodology for personality evaluation. Oxford, England: Ronald Press.

Lewin, K. (1935). A dynamic theory of personality. New York: McGraw-Hill.

Locke, K. D. (2000). Circumplex scales of interpersonal values: Reliability, validity, and applicability to interpersonal problems and personality disorders. Journal of Personality Assessment, 75, 249-267.

MacLeod, C. (1999). Anxiety and anxiety disorders. In: T. Dalgleish \& M. J. Power (Eds.), Handbook of cognition and emotion (pp. 447-477). New York: John Wiley \& Sons.

Mathews, A. (2012). Personality and information processing: We are what we encode. European Journal of Personality, 26, 158-163.

Mathews, A., \& MacLeod, C. (2005). Cognitive vulnerability to emotional disorders. Annual Review of Clinical Psychology, 1, 167-195.

McAdams, D. P., Hoffman, B. J., Mansfield, E. D., \& Day, R. (1996). Themes of agency and communion in significant autobiographical scenes. Journal of Personality, 64, 339-377.

McClelland, D. (1987). Human motivation. New York: Cambridge University Press.

Miller, G. (2000). The mating mind: How sexual choice shaped the evolution of human nature. New York: Doubleday \& Co.

Miller, N. E. (1944). Experimental studies of conflict. In J. V. Hunt (Ed.), Personality and the behavior disorders (pp. 431-465). Oxford, England: Ronald Press. 
Miyake, A., Friedman, N. P., Emerson, M. J., Witzki, A. H., \& Howerter, A. (2000). The unity and diversity of executive functions and their contributions to complex 'frontal lobe' tasks: A latent variable analysis. Cognitive Psychology, 41, 49-100.

Mogg, K., \& Bradley, B. P. (1998). A cognitive-motivational analysis of anxiety. Behaviour Research and Therapy, 36, 809-848.

Moskowitz, D. (1994). Cross-situational generality and the interpersonal circumplex. Journal of Personality and Social Psychology, 66, 921-933.

Myers, D. G., \& Diener, E. (1995). Who is happy? Psychological Science, 6, 10-19.

Ode, S., Winters, P. L., \& Robinson, M. D. (2012). Approach motivation as incentive salience: Perceptual sources of evidence in relation to positive word primes. Emotion, 12, 91-101.

Pervin, L. A. (1994). A critical analysis of current trait theory. Psychological Inquiry, 5, 103113.

Proffitt, D. R. (2006). Embodied perception and the economy of action. Perspectives on Psychological Science, 1, 110-122.

Rauthmann, J. F., \& Kolar, G. P. (2013). Positioning the Dark Triad in the interpersonal circumplex: The friendly-dominant narcissist, hostile-submissive Machiavellian, and hostile-dominant psychopath? Personality and Individual Differences, 54, 622-627.

Robinson, M. D. (2004). Personality as performance: Categorization tendencies and their correlates. Current Directions in Psychological Science, 13, 127-129.

Robinson, M. D. (2007). Lives lived in milliseconds: Using cognitive methods in personality research. In: R. W. Robins, R. C. Fraley, \& R. Krueger (Eds.), Handbook of research methods in personality psychology (pp. 345-359). New York: Guilford Press. 
Robinson, M. D., \& Gordon, K. H. (2011). Personality dynamics: Insights from the personality social cognitive literature. Journal of Personality Assessment, 93, 161-176.

Robinson, M. D., \& Liu, T. (2013). Perceptual negativity predicts greater reactivity to negative events in daily life. Personality and Individual Differences, 55, 926-930.

Robinson, M. D., Moeller, S. K., \& Ode, S. (2010). Extraversion and reward-related processing: Probing incentive motivation in affective priming tasks. Emotion, 10, 615-626.

Robinson, M. D., \& Neighbors, C. (2006). Catching the mind in action: Implicit methods in personality research and assessment. In: M. Eid \& E. Diener (eds.), Handbook of multimethod measurement in psychology (pp. 115-125). Washington, DC: American Psychology Association.

Robinson, M. D., \& Wilkowski, B. M. (in press). Personality processes and processes as personality: A cognitive perspective. In: M. Mikulincer \& P. R. Shaver (Eds.), APA handbook of personality and social psychology. Washington, DC: American Psychological Association.

Robinson, M. D., Zabelina, D. L., Boyd, R. L., Bresin, K., \& Ode, S. (in press). The self’s symbolic role in implicit approach/avoidance: Movement time evidence. The Journal of Social Psychology.

Robinson, T. E., \& Berridge, K. C. (2003). Addiction. Annual Review of Psychology, 54, 25-53.

Ruiz, J. M., Smith, T. W., \& Rhodewalt, F. (2001). Distinguishing narcissism and hostility: Similarities and differences in interpersonal circumplex and five-factor correlates. Journal of Personality Assessment, 76, 537-555. 
Smith, T. W., Glazer, K., Ruiz, J. M., \& Gallo, L. C. (2004). Hostility, anger, aggressiveness, and coronary heart disease: An interpersonal perspective on personality, emotion, and health. Journal of Personality, 72, 1217-1270.

Smith, T. W., Traupman, E. K., Uchino, B. N., \& Berg, C. A. (2010). Interpersonal circumplex descriptions of psychosocial risk factors for physical illness: Application to hostility, neuroticism, and marital adjustment. Journal of Personality, 78, 1011-1036.

Sullivan, H. S. (1953). Conceptions of modern psychiatry. New York: W. W. Norton \& Co.

Tamir, M., \& Robinson, M. D. (2007). The happy spotlight: Positive mood and selective attention to rewarding information. Personality and Social Psychology Bulletin, 33, 1124-1136.

Trapnell, P. D., \& Paulhus, D. L. (2012). Agentic and communal values: Their scope and measurement. Journal of Personality Assessment, 94, 39-52.

Trobst, K. K. (2000). An interpersonal conceptualization and quantification of social support transactions. Personality and Social Psychology Bulletin, 26, 971-986.

Veltkamp, M., Aarts, H., \& Custers, R. (2008). Perception in the service of goal pursuit: Motivation to attain goals enhances the perceived size of goal-instrumental objects. Social Cognition, 26, 720-736.

Wiggins, J. S. (1979). A psychological taxonomy of trait-descriptive terms: The interpersonal domain. Journal of Personality and Social Psychology, 37, 395-412.

Wiggins, J. S. (1991). Agency and communion as conceptual coordinates for the understanding and measurement of interpersonal behavior. In: D. Cicchetti \& W. M. Grove (Eds.), Thinking clearly about psychology: Essays in honor of Paul E. Meehl (pp. 89-113). Minnesota, MN: University of Minnesota Press. 
Wiggins, J. S., \& Broughton, R. (1991). A geometric taxonomy of personality scales. European Journal of Personality, 5, 343-365.

Wiggins, J. S., \& Trapnell, P. D. (1996). A dyadic-interactional perspective on the five-factor model. In J. S. Wiggins, J. S. Wiggins (Eds.), The five-factor model of personality: Theoretical perspectives (pp. 88-162). New York: Guilford Press.

Wiggins, J. S., Trapnell, P., \& Phillips, N. (1988). Psychometric and geometric characteristics of the Revised Interpersonal Adjective Scales (IAS-R). Multivariate Behavioral Research, $23,517-530$.

Wilkowski, B. M., \& Robinson, M. D. (2007). Keeping one's cool: Trait anger, hostile thoughts, and the recruitment of limited capacity control. Personality and Social Psychology Bulletin, 33, 1201-1213.

Wilkowski, B. M., \& Robinson, M. D. (2008). Guarding against hostile thoughts: Trait anger and the recruitment of cognitive control. Emotion, 8, 578-583.

Wilkowski, B. M., \& Robinson, M. D. (2010). The anatomy of anger: An integrative model of trait anger and reactive aggression. Journal of Personality, 78, 9-38.

Wurm, L., \& Vakoch, D. (2000). The adaptive value of lexical connotation in speech perception. Cognition and Emotion, 14, 177-191.

Wyvell, C. L., \& Berridge, K. C. (2000). Intra-accumbens amphetamine increases the conditioned incentive salience of sucrose reward: Enhancement of reward 'wanting' without enhanced 'liking' or response reinforcement. The Journal of Neuroscience, 20, $8122-8130$. 


\section{Figure 1}

Estimated Categorization Speed Means by Cue Type (Power versus Affiliation) and

Interpersonal Arrogance (Low = -1 SD; High = +1 SD), Study 1

Figure 2

Estimated Font Size Overestimation Means by Cue Type (Power versus Affiliation) and

Interpersonal Arrogance (Low = -1 SD; High = +1 SD), Study 2

Figure 3

Estimated Movement Time Means by Cue Type (Power versus Affiliation) and Movement

Direction (Approach versus Avoid) at a High (+1 SD) Level of Interpersonal Arrogance, Study 3 